Contributions to Game Theory and Management, XIII, 335-346

\title{
Search Strategies in the Game with a Team of Pursuers and One Evader on the Plane
}

\author{
Semyon V. Mestnikov and Nikolay V. Petrov \\ North-Eastern Federal University, \\ Institute of Mathematics and Informatics, \\ Belinskogo Street 58, Yakutsk, 677000, Russia \\ E-mail: mestsv@mail.ru, stalker_pnv@mail.ru
}

\begin{abstract}
We consider a differential simple search game without phase constraints on the plane. Each pursuer from the team chooses an initial position and piecewise constant strategy. As an example, the game of simple search of immobile hider (evader) on the plane is considered, and the necessary conditions for detection are found. For cases with two and three pursuers, a comparison of the detection time of the evader was made and approximate information sets numerically constructed.
\end{abstract}

Keywords: differential search game, information set, the pursuer team, numerical construction, comparison of detection time.

\section{Introduction}

In this paper, we consider the zero-sum differential search game (Petrosjan and Zenkevich, 1986; Zenkevich and Mestnikov, 1991; Petrosjan and Garnaev, 1992) from the pursuer viewpoint. The research relates to studies (Mestnikov, 1994; Mestnikov, 1996; Mestnikov and Petrov, 2017), in which the properties and problems of information sets approximation for various classes of specific differential search games were examined both from the side of the pursuer and from the side of the evading player.

Currently, definition, construction, and approximation of information sets of players are among the important tasks of the differential game theory (links in the paper The Hohzaki, 2016). The method of approximation and numerical construction of information sets was described in (Mestnikov, Petrov and Everstova, 2014).

In this work, we present a search game with evader $E$ and pursuers team $\bar{P}$. For some cases, when the pursuers team $\bar{P}$ consists of two or three players, sufficient conditions were found on the parameters of the game to guarantee the detection of player $E$ in one class of strategies. A search time comparison was also performed for these strategies.

Using a computer program, we numerically constructed information sets for the games in question. 


\section{The Game}

The zero-sum differential search game between team of pursuers $\bar{P}=\left\{P_{1}, \ldots, P_{k}\right\}$ and evader $E$ is considered. The following differential equation describes the dynamic of the game

$$
\begin{gathered}
P_{i}: \dot{x}_{i}=u_{i},\left|u_{i}\right| \leq \alpha, x(0)=x_{0},\left|x_{0}\right|=r+l, x_{i}, u_{i} \in R^{2}, \\
E: \dot{y}=v,|v| \leq \beta, y(0)=y_{0},\left|y_{0}\right| \leq r, \beta \leq \alpha, y, v \in R^{2},
\end{gathered}
$$

where $i=1, \ldots, k, x_{0}$ - location of pursuer $P_{i}$ at time point $t_{0}=0, y_{0}$ - location of evader $E$ at time point $t_{0}=0, \alpha, \beta, r, l$ - game parameters.

The detection set $S(x)$ of player $P_{i}, i=1, \ldots, k$ is the disk of radius $l$ centered at the position of the pursuer. We consider simple search game between evader $E$ and team $\bar{P}=\left\{P_{1}, \ldots, P_{k}\right\}$ of similar pursuers acting like one.

The admissible controls $u_{i}=u_{i}(t), t \geq 0$, player $P_{i}, i=1, \ldots, k$ - piecewise constant functions with two intervals of constancy. The set all admissible controls of player $P_{i}, i=1, \ldots, k$ we will denote as $D_{P}$. Under pure strategy $a_{i}$ of a player $P_{i}, i=1, \ldots, k$ will be denoted as pair $a_{i}=\left(x_{0}^{*}, u_{i}(\cdot)\right)$, where the point $x_{0}^{*} \in R^{2}$ satisfies the condition (1).

The admissible controls $v=v(t), t \geq 0$, of a player $E$ - are piecewise continuous functions. The set of all admissible controls of a player $E$ is denoted as $D_{E}$. Under pure strategy $b$ of a player $E$ will be denoted as pair $b=\left(y_{0}^{*}, v(\cdot)\right)$, where $y_{0}^{*} \in R^{2}$ satisfies the condition (2). The game is considered in program strategies.

The state of information in the game is as follows. In the initial moment of time $t=t_{0}$, player $P_{i}(E), i=1, \ldots, k$ possesses only a priori information about another player, that is, he knows $\left\|y_{0}^{*}\right\| \leq r\left(\left\|x_{0}^{*}\right\|=r+l\right)$ and game dynamics (1),(2). In the further game process, players do not receive current information about the location of the enemy.

The game proceeds as follows: player $E$ selects some point $y_{0}^{*}$ from a circle of radius $r$ and admissible controls $v(\cdot)$. Player $P_{i}$ chooses the strategy $a_{i}=\left(x_{0}^{*}, u_{i}(\cdot)\right)$, not knowing the strategy of player $E$. Player $E$ considered detected (caught) at time moment $t$, if distance between $x_{i}(t), i=1, \ldots, k$ and $y(t)$ is less or equal to $l$, where $l$ - a given positive number.

Assume that for each state $x_{i} \in R^{2}, i=1, \ldots, k$ is defined the set $S(x) \subset R^{2}$ closed circle of radius $l$ with center in the point $x$, which in the future will be called detection domain player $P_{i}, i=1, \ldots, k$. Then $\bar{a}=\left(\left(x_{0}, u_{1}(\cdot)\right), \ldots,\left(x_{0}, u_{k}(\cdot)\right)\right)$ is called strategies for pursuers team $\bar{P}$. Winning Function of team $\bar{P}$ is defined as in the paper(Mestnikov and Petrov, 2017).

\section{The Game, if Pursuers Team $\bar{P}$ Consists of One Player}

Assume that for the pursuer team $\bar{P}=\left\{P_{1}, \ldots, P_{k}\right\}, k=1$ for $\beta=0$ (immobile hider). In other words, pursuer team consists of only one player $P$, who at initial time $t_{0}=0$ is at the point $x_{0}$ and selects control with two intervals of constancy.

$$
u(\cdot)= \begin{cases}u_{1}, & 0<t \leq t^{*}, \\ u_{2}, & t^{*}<t<\infty\end{cases}
$$


where

$$
u_{1}=\left(\begin{array}{l}
u_{11} \\
u_{12}
\end{array}\right), u_{2}=\left(\begin{array}{l}
u_{21} \\
u_{22}
\end{array}\right)
$$

that is, the strategies of player $P$ are piecewise constant strategies $a=\left(x_{0}^{*}, u_{i}(\cdot)\right)$. The trajectory generated by the strategy $a=\left(x_{0}^{*}, u(\cdot)\right)$ is denoted by $x(\cdot)=$ $x\left(\cdot, t_{0}, x_{0}^{*}, u(\cdot)\right)$. Such a trajectory on the plane is represented as the union of the segment $\left[x_{0}^{*} ; x\left(t^{*}\right)\right]$ and a ray with beginning at point $x\left(t^{*}\right)$ or a polyline with two links (Fig. 1).

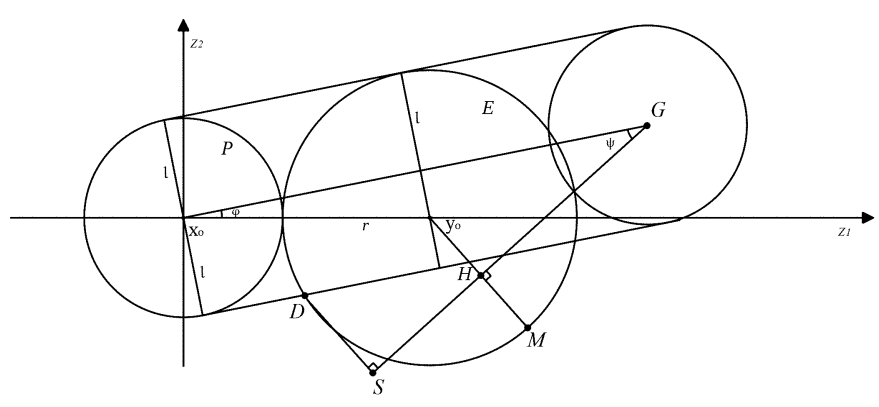

Fig. 1. Game with one pursuer player $P$.

The time instant $t^{*}$ will be called the turning moment. In this case, the first peak of a broken line is at point $x_{0}^{*}$, the second peak at point $G=x\left(t^{*}\right)$ at the time $t^{*}$ and third at the point $S=x(T)$. The case when $u(\cdot)=$ const, with one interval, was described in (Mestnikov and Petrov, 2017).

Represent now this game on the plane. To do this, we draw a rectangular Cartesian coordinate system so that the initial position $x_{0}^{*}$ of player $P$ coincides with the origin of coordinates $O z_{1} z_{2}$, and the center of the initial location of player $E$, a circle of radius $r$, with center on the axis $O z_{1}$.

Further, we will denote the initial point $x_{0}^{*}$ by $x_{0}$, and the center of the circle of radius $\mathrm{r}$ of the initial location of player $E$ by $y_{0}$.

The distance between points $x_{0}$ and $y_{0}$ is $r+l$.

$$
x_{0}=\left(\begin{array}{l}
0 \\
0
\end{array}\right), \quad y_{0}=\left(\begin{array}{c}
r+l \\
0
\end{array}\right) .
$$

By $T$, we denote the total game time for which the pursuer $P$ is guarantees the capture of player $E$. If the inequality $r<2 l$ is fulfilled, the pursuer $P$ guarantees the capture of evader $\mathrm{r} E$ in a finite time. It is clear that $r \rightarrow 2 l(r<2 l)$ then $T \rightarrow \infty$.

In case when $T<\infty$, the total game duration $T$ can be minimized by choosing the optimal turning moment $t^{*}$ and control $u(\cdot)$ (Fig. 2).

The conditions for the parameters of the game for guaranteed detection, numerical optimization of turn time, and numerical construction of information sets in a game with one pursuer player $P_{1}$ were considered in (Mestnikov and Petrov, 2018). 


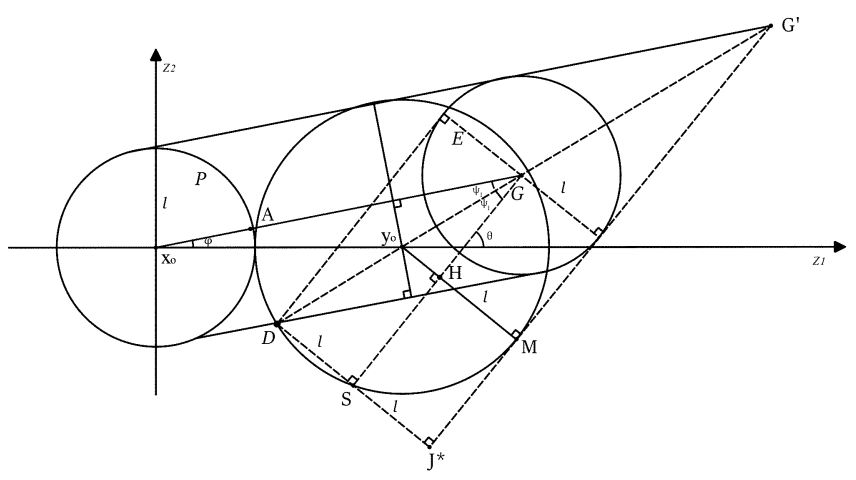

Fig. 2. Guaranteed Player $E$ Detection at Optimal Turn Time $t$

\section{The Game, if Pursuers Team $\bar{P}$ Consist of Several Players}

If $r>2 l$ of the pursuer $\mathrm{P}$ one player cannot guarantee the capture of player $E$. Next, we consider a game with the team $\bar{P}=\left\{P_{1}, \ldots, P_{k}\right\}, k=2$ for $\beta=0$.

We define two piecewise constant strategies for the pursuer team $\bar{P}=\left\{P_{1}, P_{2}\right\}$, where the initial position $x_{0}$ at the time $t_{0}=0$ is the same.

$$
x_{0}=\left(\begin{array}{c}
-2 \sqrt{r l} \\
r-l
\end{array}\right), \quad y_{0}=\left(\begin{array}{l}
0 \\
0
\end{array}\right) .
$$

To do this, denote by $x_{i}(1, l+), i=1,2$, a straight line parallel and lying above (to the left of the direction of movement) at a distance $l$ from the trajectory of the first pursuer $P_{i}$, in case $0 \leq t \leq t^{*}, x_{i}(2, l+)-$ at $t^{*}<t<\infty$.

And through $x_{i}(1, l-)$, a straight line parallel and lying below (to the right of the direction of movement) at a distance $l$ from the trajectory of the first pursuer player $P_{i}$, in the case $0 \leq t \leq t^{*}, x_{i}(2, l-)-$ at $t^{*}<t<\infty$.

We assume that the first pure strategy of the pursuer team $\bar{P}$ is the strategy $\bar{a}_{1}=\left(a_{11}, a_{12}\right)$, where $a_{11}=\left(x_{0}, u_{1}(\cdot)\right)$ and $a_{12}=\left(x_{0}, u_{2}(\cdot)\right)$.

We assume that the second pure strategy of a team of pursuers $\bar{P}$ is the strategy $\bar{a}_{2}=\left(a_{21}, a_{22}\right)$, where $a_{21}=\left(x_{0}, u_{3}(\cdot)\right)$ and $a_{22}=\left(x_{0}, u_{3}(\cdot)\right)$.

We define the motion of players according to these strategies.

Definition 1. Strategy $a_{1}$ for the pursuers team $\bar{P}=\left\{P_{1}, P_{2}\right\}$ is the strategy where the first pursuer $P_{1}$ chooses control $u_{1}(\cdot)$

$$
u_{1}(\cdot)= \begin{cases}u_{11}, & 0<t \leq t^{*} \\ u_{12}, & t^{*}<t<\infty\end{cases}
$$

where

$$
u_{11}=\left(\begin{array}{c}
\cos \varphi_{1} \\
\sin \varphi_{1}
\end{array}\right), u_{12}=\left(\begin{array}{c}
\cos \psi_{1} \\
\sin \psi_{1}
\end{array}\right),
$$

the angle $\varphi_{1}$ is chosen so that the line $x_{1}(1, l+)$ touches the circle of uncertainty of the evader $E$ (the circle of radius $r$ centered at $y_{0}$ ). The second angle $\psi_{1}$ is chosen 
so that the straight line $x_{1}(2, l+)$ passes through point $D$, where the point $D$ is the first intersection point of the straight line $x_{1}(1, l-)$ of the circle of radius $r$ centered at the point $y_{0}($ Fig $3(\mathrm{a}))$.

The second pursuer $P_{2}$ selects $u_{2}(\cdot)$

$$
u_{2}(\cdot)= \begin{cases}u_{21}, & 0<t \leq t^{*} \\ u_{22}, & t^{*}<t<\infty\end{cases}
$$

where

$$
u_{21}=\left(\begin{array}{c}
\cos \varphi_{2} \\
\sin \varphi_{2}
\end{array}\right), u_{22}=\left(\begin{array}{c}
\cos \psi_{2} \\
\sin \psi_{2}
\end{array}\right)
$$

the angle $\varphi_{2}$ is chosen in such a way that the line $x_{2}(1, l+)$ passes through point $B$, where $B$ is first intersection point of the line $x_{1}(2, l-)$ with a circle of radius $r$ centered at $y_{0}$. The second angle $\psi_{2}$ is chosen in such a way that the line $x_{2}(2, l+)$ passes through the point $C$ or $Z$, where $C$ is the second intersection point of the line $x_{1}(2, l-)$ with the circle of radius $r$ centered at $y_{0}$, and $Z$ is the intersection point of the line $x_{1}(2, l-)$ and line $x_{2}(1, l-)$. Moreover, if the point $Z$ lies lower than the straight line $x_{2}(2, l+)$ passing through the point $C$, then the straight line $x_{2}(2, l+)$ passing through the point $C$ is selected; otherwise, the straight line $x_{2}(2, l+)$ passing through the point $Z$ is selected (Fig $3(\mathrm{~b})$ ).

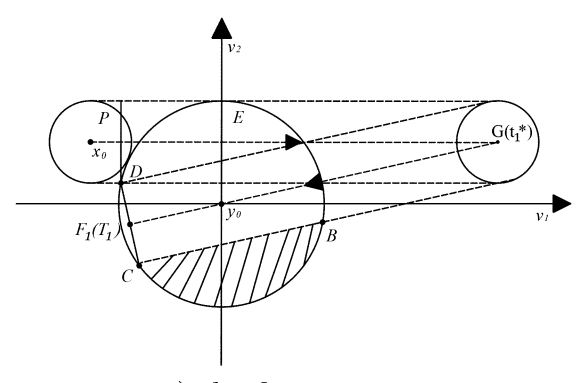

a) the first pursuer

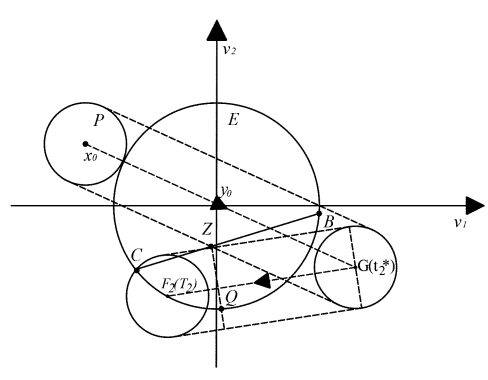

b) the second pursuer

Fig. 3. First Strategy

Definition 2. The strategy $a_{2}$ for the pursuer team $\bar{P}=\left\{P_{1}, P_{2}\right\}$ is the strategy where the first pursuer player $P_{1}$ chooses control $u_{3}(\cdot)$

$$
u_{3}(\cdot)= \begin{cases}u_{31}, & 0<t \leq t^{*} \\ u_{32}, & t^{*}<t<\infty\end{cases}
$$

where

$$
u_{31}=\left(\begin{array}{c}
\cos \varphi_{1} \\
\sin \varphi_{1}
\end{array}\right), u_{32}=\left(\begin{array}{c}
\cos \psi_{1} \\
\sin \psi_{1}
\end{array}\right),
$$

the angle $\varphi_{1}$ is chosen so that the line $x_{1}(1, l+)$ touches the circle of the uncertainty of the evader $E$ (the circle of radius $r$ centered at $y_{0}$ ). The second angle $\psi_{1}$ is chosen so that the line $x_{1}(2, l+)$ also touches the uncertainty circle of the evader $E$ (Fig $4(\mathrm{a}))$.

The second pursuer $P_{2}$ selects control $u_{4}(\cdot)$ 


$$
u_{4}(\cdot)= \begin{cases}u_{41}, & 0<t \leq t^{*} \\ u_{42}, & t^{*}<t<\infty\end{cases}
$$

where

$$
u_{41}=\left(\begin{array}{c}
\cos \varphi_{2} \\
\sin \varphi_{2}
\end{array}\right), u_{42}=\left(\begin{array}{c}
\cos \psi_{2} \\
\sin \psi_{2}
\end{array}\right),
$$

the angle $\varphi_{2}$ is chosen in such a way that the line $x_{2}(1, l+)$ passes through point $B_{1}$ or $Z$, where $B_{1}$ is the second intersection point of the line $x_{1}(1, l-)$ of a circle of radius $r$ centered at $y_{0}, Z$ is the intersection point of the line $x_{1}(1, l-)$ and the line $x_{1}(2, l+)$. Moreover, if the point $Z$ is outside the circle of radius $r$ centered at the point $y_{0}$, then the angle $\varphi_{2}$ is chosen so that the straight line $x_{2}(2, l+)$ passes through the point $B_{1}$, otherwise, $\varphi_{2}$ so that the straight line $x_{2}(2, l+)$ passes through the point $Z$ (Fig.4(b)).

The second angle $\psi_{2}$ is chosen in such a way that the line $x_{2}(2, l+)$ passes through point $D$, where $D$ is the second intersection point of the line $x_{1}(2, l-)$ of a circle of radius $r$ centered at $y_{0}(\mathrm{Fig} 4(\mathrm{~b}))$.

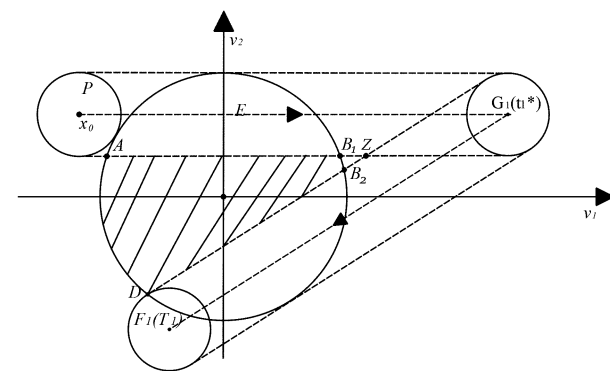

a) the first pursuer

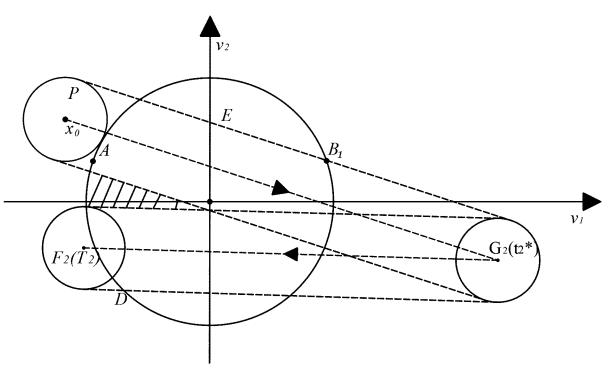

b) the second pursuer

Fig. 4. The Second Strategy

\section{Sufficient Conditions for Guaranteed Detection of Strategies $a_{1}$ and $a_{2}$}

For cases when the total search time for the pursuers team $\bar{P}=\left\{P_{1}, \ldots, P_{k}\right\}$, $k=2$ for $\beta=0$ is unlimited $T \rightarrow \infty$, strategies $a_{1}$ and $a_{2}$ will look as follows (Fig 5 , Fig 6).

Further, we analytically find sufficient conditions for guaranteed detection of the evader $E$ by pursuer team $\bar{P}$ for strategies $a_{1}$ and $a_{2}$ for $\beta=0$ (immobile hider). Search games for $\beta>0$ are investigated only numerically, but not described in this article.

We find sufficient conditions for the strategy $a_{1}$. For this, it is necessary to find the angular coefficient of the line $\tan \varphi$ passing through the point $C\left(4 \sqrt{r l / 2+l^{2}} ; r-\right.$ $4 l$ ) and being tangent to the circle of radius $l$ centered at $x_{0}(-2 \sqrt{r l} ; r-l)($ Fig $5(\mathrm{~b}))$ using the formula

$$
l=\frac{\left|-2 \tan \varphi \sqrt{r l}-4 \tan \varphi \sqrt{r l / 2+l^{2}}+3 l\right|}{\sqrt{\tan ^{2} \varphi+1}}
$$




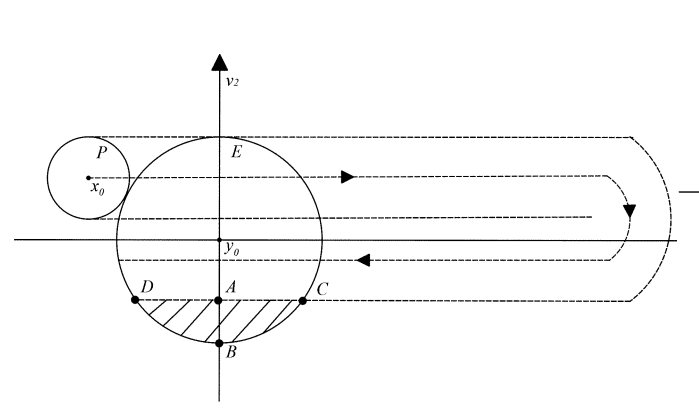

a) the first pursuer

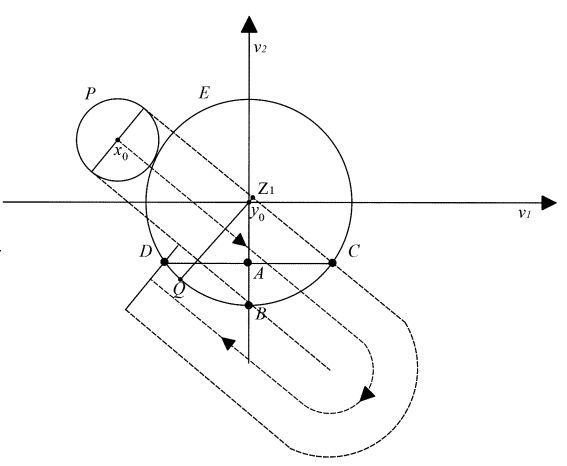

b) the second pursuer

Fig. 5. First Strategy pursuers team with infinite time

To find the coordinates of the point $Z_{1}$ we use the system

$$
\left\{\begin{array}{l}
v_{2}=\tan \varphi\left(v_{1}-C_{v_{1}}\right)+C_{v_{2}} \\
v_{2}=-\frac{v_{1}}{\tan \varphi}
\end{array}\right.
$$

The coordinates of the point $Q$ are the solutions of the system corresponding to the schedule

$$
\left\{\begin{array}{l}
r^{2}=v_{1}^{2}+v_{1}^{2} \\
v_{2}=-\frac{v_{1}}{\tan \varphi}
\end{array}\right.
$$

Thus, the guaranteed detection of an evader $E$ in a finite time $T$ by a pursuer team $\bar{P}$ is performed provided that the distance $\left|Q Z_{1}\right|<4 l$.

To find a sufficient condition for detecting an evader $E$ when players $\bar{P}=$ $\left\{P_{1}, \ldots, P_{k}\right\}$ choose a strategy $a_{2}$, you need to find the angular coefficient of the line passing through the point $B\left(2 \sqrt{r l-l^{2}} ; r-2 l\right)$, which is also tangent to a circle of radius $l$ centered at $x_{0}(-2 \sqrt{r l} ; r-l)($ Fig $6(\mathrm{~b}))$.

$$
l=\frac{\left|-2 \tan \varphi \sqrt{r l}-2 \tan \varphi \sqrt{r l-l^{2}}-l\right|}{\sqrt{\tan ^{2} \varphi+1}}
$$

To find the coordinates of the point $Z_{2}$ we use the system

$$
\left\{\begin{array}{l}
v_{2}=\tan \varphi\left(v_{1}-B_{v_{1}}\right)+B_{v_{2}} \\
v_{2}=-\frac{\left(v_{1}-D_{v_{1}}\right)}{\tan \varphi}+D_{v_{2}}
\end{array}\right.
$$

where are the coordinates of the point are $D\left(-2 \sqrt{r l-l^{2}} ; 2 l-r\right)$.

Thus, the guaranteed detection of an evader $E$ by a search of pursuer players $\bar{P}$ for a finite time when choosing the strategy $a_{2}$ is performed under the condition that the distance $\left|Q Z_{2}\right|<4 l$. 


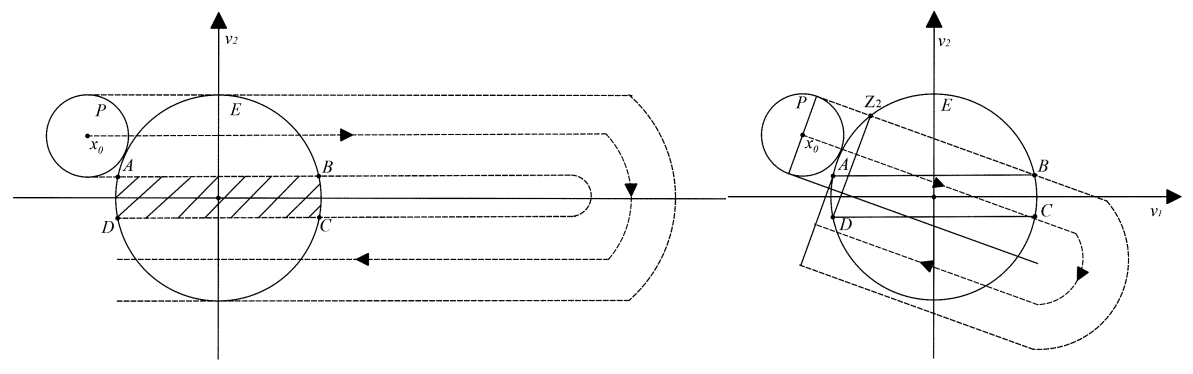

a) the first pursuer $\quad$ b) the second pursuer

Fig. 6. Second Strategy pursuers team with infinite time

\section{Numerical Comparison of the Guaranteed Detection of Strategies $a_{1}$ and $a_{2}$ with the Same Game Parameters}

We construct numerically informational sets with a pursuers team $\bar{P}=\left\{P_{1}, \ldots, P_{k}\right\}$ for $k=2$ and $k=3$, using the developed computer program.

\subsection{Total Time of Guaranteed Detection of Strategy $a_{1}$}

To select the strategy $a_{1}$ for pursuers team $\bar{P}=\left\{P_{1}, \ldots, P_{k}\right\}, k=2$, it is necessary for each player to set the turn time $t^{*}$ (Fig.7).

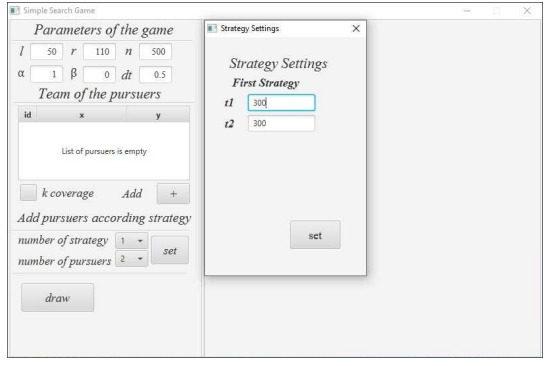

a) Define turn time

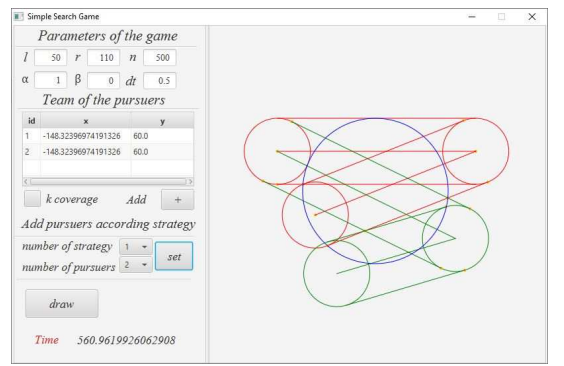

b) Build trajectory $a_{1}$

Fig. 7. Setting initial parameters for the game when $k=2$

After constructing the trajectory of players from the team $P$, the total time of the game $T$ will be calculated To check the guaranteed detection of an evader $E$ by pursuers team $\bar{P}$, it is necessary to start the game (Fig.8).
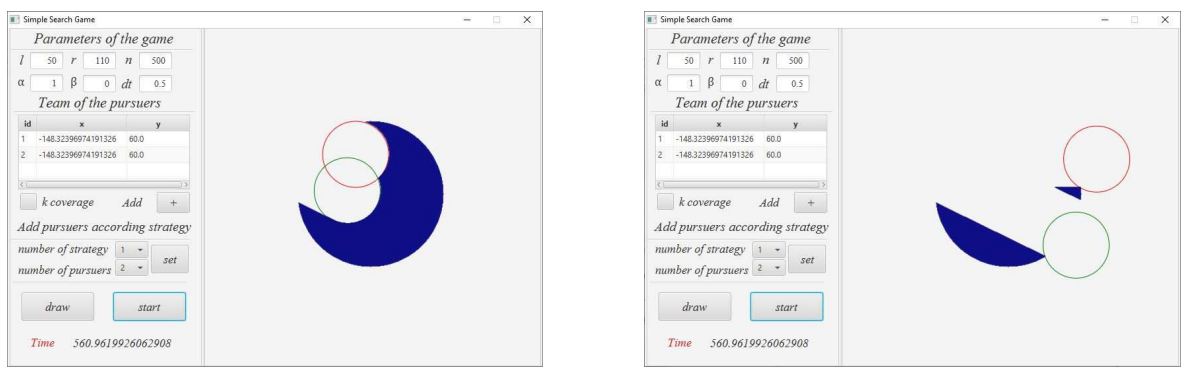

Fig. 8. Movement of pursuers $\bar{P}$ on selection $a_{1}$ 
Consider a search game with pursuer team $\bar{P}=\left\{P_{1}, \ldots, P_{k}\right\}, k=3$ (Fig.9, Fig.10).

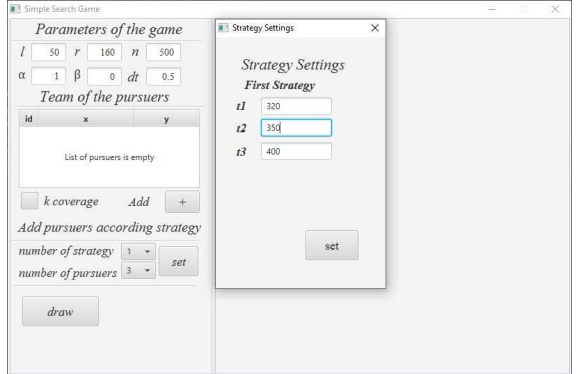

a) Define turn time

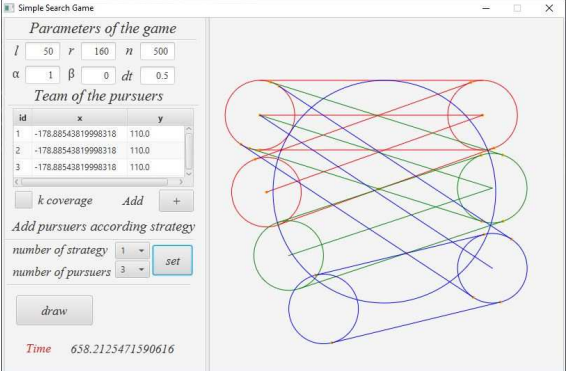

b) Build trajectory $a_{1}$

Fig. 9. Setting initial parameters for the game when $k=3$
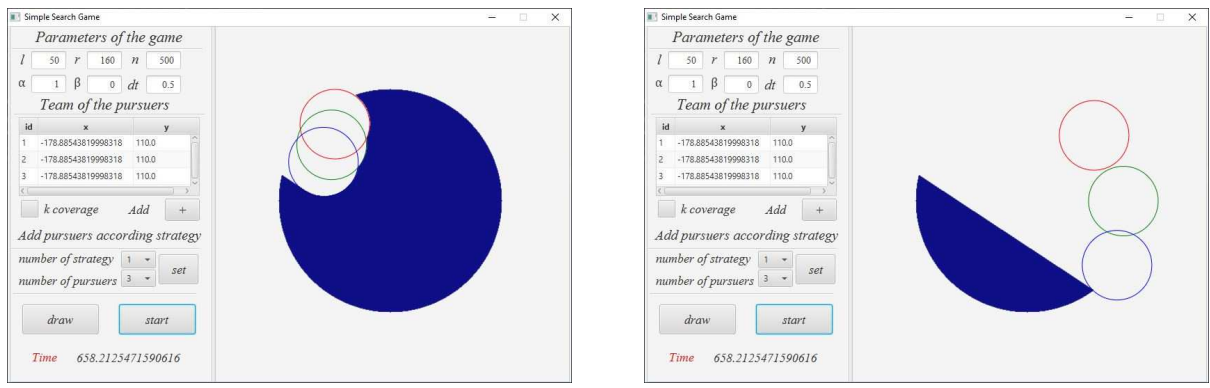

Fig. 10. Game with pursuers team $\bar{P}$ when $k=3$

\subsection{Total Guaranteed Detection Time of Strategy $a_{2}$}

To select the strategy $a_{2}$ for the pursuer team $\bar{P}=\left\{P_{1}, \ldots, P_{k}\right\}, k=2$, it is necessary for each player to set the turn time $t^{*}$ (Fig.11).

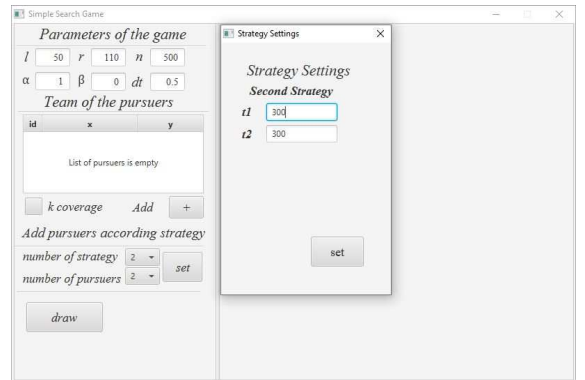

a) Define turn time

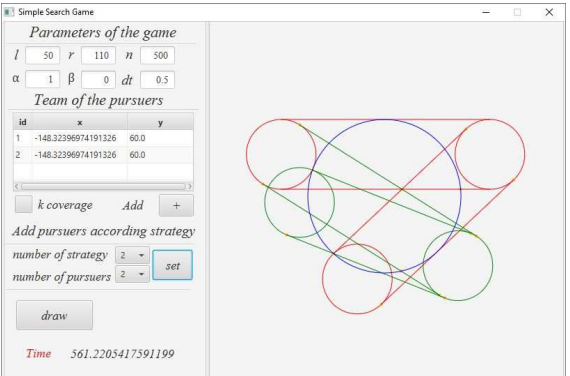

b) Build trajectory $a_{2}$

Fig. 11. Setting initial parameters for the game when $k=2$

After constructing the trajectory of players from team $P$, the total time of the game $T$ will be calculated To check the guaranteed detection of an evader $E$ by pursuer team $\bar{P}$ you must start the game (Fig.12). 

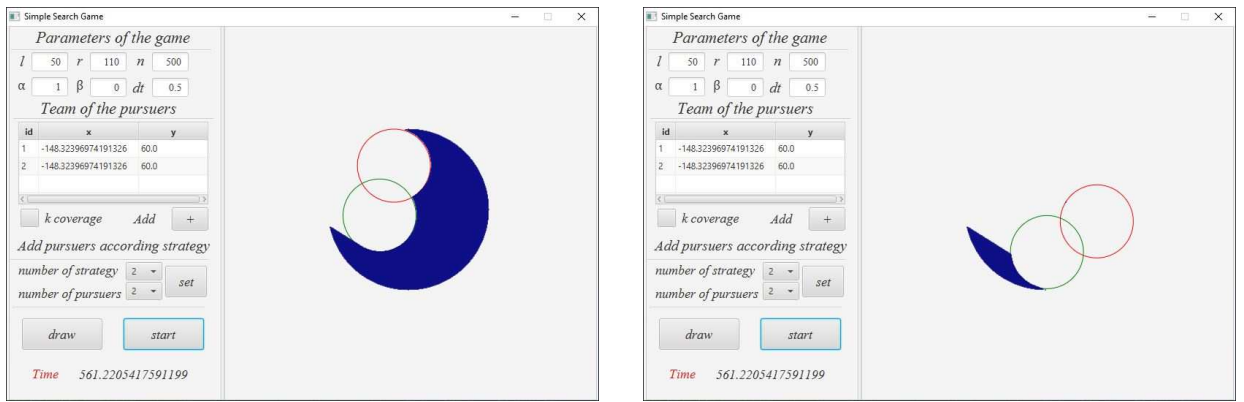

Fig. 12. Movement of pursuers $\bar{P}$ on selection $a_{2}$

Consider search game with pursuer team $\bar{P}=\left\{P_{1}, \ldots, P_{k}\right\}, k=3$ (Fig.13, Fig.14).

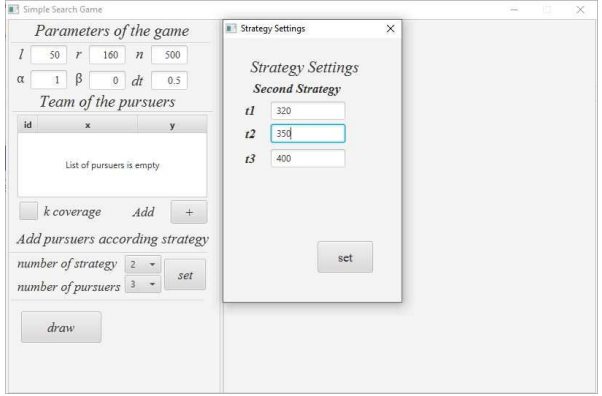

a) Define turn time

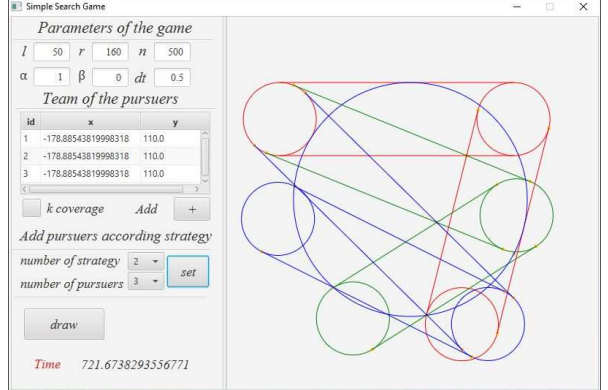

b) Build trajectory $a_{2}$

Fig. 13. Setting initial parameters for the game when $k=3$
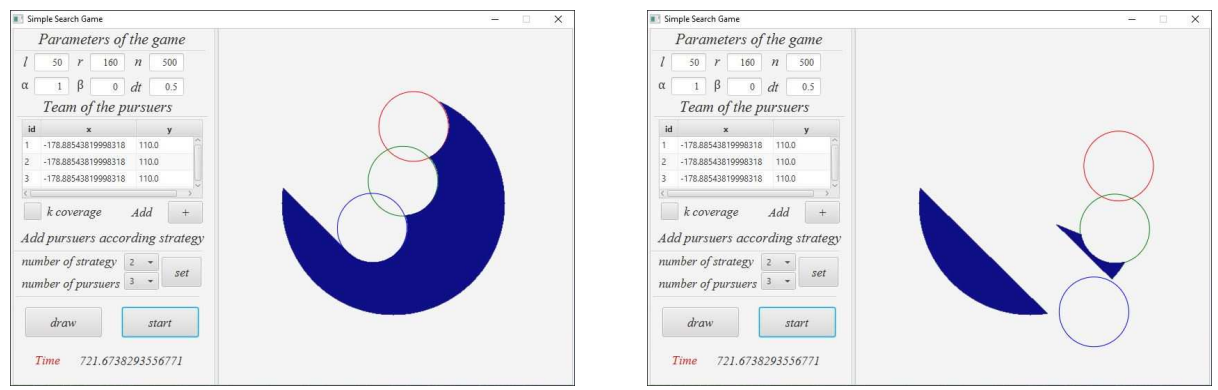

Fig. 14. Game with pursuer team $\bar{P}$ when $k=3$

\subsection{Comparison of Total Time for Strategies $a_{1}$ and $a_{2}$}

Let us numerically compare the detection time for $a_{1}$ and $a_{2}$ for the same rotation time $t^{*}$ of the pursuer team $\bar{P}=\left\{P_{1}, \ldots, P_{k}\right\}$ for $k=2$ and game parameters $r, l$, $\alpha, \beta$ (Fig.15).

The histogram shows a comparison of the detection time of an evader $E$ by pursuer team $\bar{P}=\left\{P_{1}, \ldots, P_{k}\right\}$ for $k=2$ for strategies $a_{1}$ and $a_{2}$ (Fig. 16). 


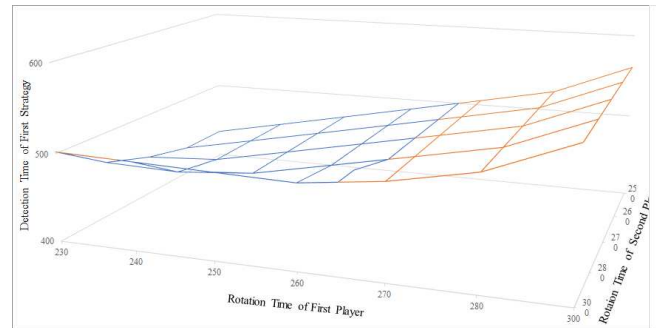

a) The First Strategy $a_{1}$

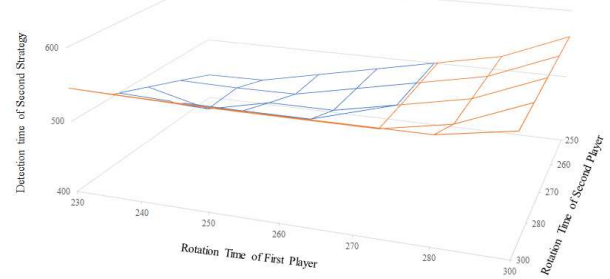

b) The Second Strategy $a_{2}$

Fig. 15. Detection time chart where $k=2$

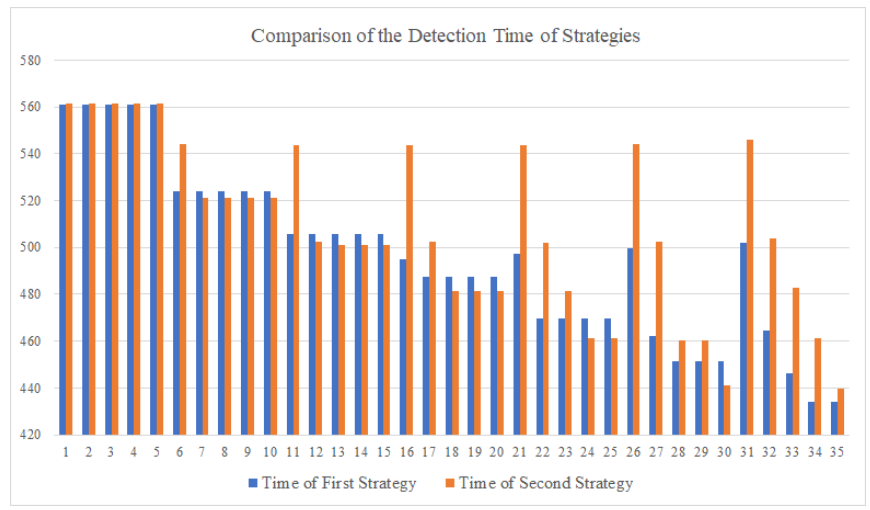

Fig. 16. Comparison of the Detection Time of two strategies when $k=2$

For the case when $\bar{P}=\left\{P_{1}, \ldots, P_{k}\right\}, k=3$, a comparison was also made of the detection time (Fig. 17).

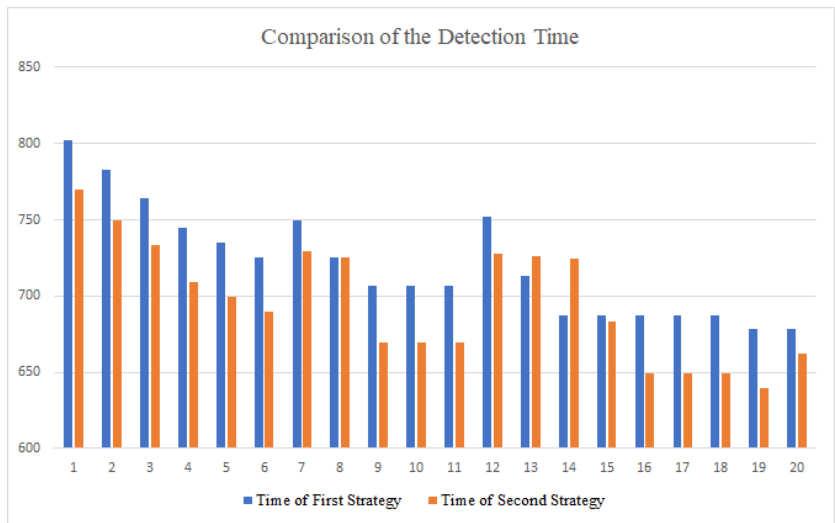

Fig. 17. Comparison of the Detection Time of two strategies when $k=3$ 


\section{Conclusion}

In the considered game of simple search on the plane with pursuer team, we compared the time of detection of an evader using two strategies. Sufficient conditions were also found for guaranteed detection of strategies in games with infinite time. The information sets for the games under consideration were numerically constructed.

Numerical calculations show that there are intervals of the values of radius of uncertainty circle of evader in which (for equal values of the remaining parameters of the game) the total detection time for first strategy is less than for second strategy and vice versa in other cases.

\section{References}

Petrosjan, L. A., Zenkevich, N. A. (1986). Optimal Search in conflict condition. St.-Peterbg. Gos. Univ.: St. Petersburg (in Russian).

Zenkevich, N. A., Mestnikov, S.V. (1991). Dynamical search of a moving object in conflict condition. Leningrad Univ., Voprosy Mekh. Protsess. Upravl., 14, 68-76: St. Petersburg (in Russian).

Petrosjan, L. A., Garnaev, A. Yu. (1992). Search games. St.-Peterbg. Gos. Univ.: St. Petersburg (in Russian).

Mestnikov, S.V. (1994). Estimates for the detection probability and uncertainty domain in a differential search game. YaGU, Math. notes of YaGU, 3, 99-104: Yakutsk (in Russian).

Mestnikov, S. V. (2002). Approximation of the information set in a differential search game with a team of pursuers. 10th international symposium on dynamic games and applications. St. Petersburg, Russia. In 2 vol. St. Petersburg: International Society of Dynamic Games, St. Petersburg State Univ., 630-631 : St. Petersburg.

Mestnikov, S. V., Petrov, N.V., Everstova, G.V. (2014). Numerical Construction of the Information Sets in the Simple Search Game with a team of pursuers and Estimates for a Detection Probability. Collected abstracts of papers presented on the Eighth International Conference Game Theory and Management / Editors Leon A. Petrosyan and Nikolay A. Zenkevich. St.Peterburg. Graduate School of Management SPbU, 207208.

Honzaki, R. (2016). Search Games: Literature and survey. J. Oper. Rec. Soc. Japan, 59(1), $1-34$.

Mestnikov, S.V., Petrov, N.V. (2017). Numerical Construction of the Information Sets and Sufficient Conditions for k-detection in the Simple Search Game on the Plane. NEFU, Mathematical Notes of NEFU, 4, 13-29: Yakutsk (in Russian).

Mestnikov, S. V., Petrov, N. V. (2018). Numerical Construction of the Information Sets and Optimization of Detection Time in the Simple Search Game with Team of Pursuers on the Plane.(English) Collected abstracts of papers presented on the Twelfth International Conference Game Theory and Management (GTM2018) / Editors Leon A. Petrosyan and Nikolay A. Zenkevich. SPb.: Graduate School of Management SPbU, 2018, 111-112. 